\title{
BMJ Open COGNITIVE-HD study: protocol of an observational study of neurocognitive functioning and association with clinical outcomes in adults with end-stage kidney disease treated with haemodialysis
}

\author{
Suetonia C Palmer, ${ }^{1}$ Marinella Ruospo, ${ }^{2,3}$ Maria Rosaria Barulli, ${ }^{4}$ Annalisa lurillo, ${ }^{4}$ \\ Valeria Saglimbene, ${ }^{2}$ Patrizia Natale, ${ }^{2}$ Letizia Gargano, ${ }^{2}$ Angelo M Murgo, ${ }^{2}$ \\ Clement Loy, ${ }^{5}$ Anita van Zwieten, ${ }^{6}$ Germaine Wong, ${ }^{6}$ Rosanna Tortelli, ${ }^{4}$ \\ Jonathan C Craig, ${ }^{6}$ David W Johnson, ${ }^{7}$ Marcello Tonelli, ${ }^{8}$ Jörgen Hegbrant, ${ }^{2}$ \\ Charlotta Wollheim, ${ }^{2}$ Giancarlo Logroscino, ${ }^{4,9}$ G F M Strippoli, ${ }^{2,6,10,11}$ \\ on behalf of the COGNITIVE-HD Study investigators
}

To cite: Palmer SC, Ruospo M, Barulli MR, et al. COGNITIVE-HD study: protocol of an observational study of neurocognitive functioning and association with clinical outcomes in adults with end-stage kidney disease treated with haemodialysis. BMJ Open 2015;5:e009328. doi:10.1136/bmjopen-2015009328

- Prepublication history for this paper is available online. To view these files please visit the journal online (http://dx.doi.org/10.1136/ bmjopen-2015-009328).

Received 7 July 2015 Revised 6 October 2015 Accepted 2 November 2015

CrossMark

For numbered affiliations see end of article.

Correspondence to Professor GFM Strippoli; gfmstrippoli@gmail.com

\section{ABSTRACT}

Introduction: The prevalence of cognitive impairment may be increased in adults with end-stage kidney disease compared with the general population. However, the specific patterns of cognitive impairment and association of cognitive dysfunction with activities of daily living and clinical outcomes (including withdrawal from treatment) among haemodialysis patients remain incompletely understood. The COGNITIVE impairment in adults with end-stage kidney disease treated with HemoDialysis (COGNITIVE-HD) study aims to characterise the age-adjusted and education-adjusted patterns of cognitive impairment (using comprehensive testing for executive function, perceptual-motor function, language, learning and memory, and complex attention) in patients on haemodialysis and association with clinical outcomes. Methods and analysis: A prospective, longitudinal, cohort study of 750 adults with end-stage kidney disease treated with long-term haemodialysis has been recruited within haemodialysis centres in Italy (July 2013 to April 2014). Testing for neurocognitive function was carried out by a trained psychologist at baseline to assess cognitive functioning. The primary study factor is cognitive impairment and secondary study factors will be specific domains of cognitive function. The primary outcome will be total mortality. Secondary outcomes will be cause-specific mortality, major cardiovascular events, fatal and non-fatal myocardial infarction and stroke, institutionalisation, and withdrawal from treatment at 12 months.

Ethics and dissemination: This protocol was approved before study conduct by the following responsible ethics committees: Catania (approval reference 186/BE; 26/09/2013), Agrigento (protocol numbers 61-62; 28/6/2013), USL Roma C (CE 39217;

\section{Strengths and limitations of this study}

Prospective study design with large sample size.

- Statistical analyses to account for age, gender, education and other potentially confounding variables.

- Mortality assessed by data linkages.

- Potential for residual confounding from measured and unmeasured variables.

24/6/2013), USL Roma F (protocol number 0041708; 23/7/2013), USL Latina (protocol number 20090/A001/ 2011; 12/7/2013), Trapani (protocol number 3413; 16/ 7/2013) and Brindisi (protocol number 40259; 6/6/ 2013). All participants have provided written and informed consent and can withdraw from the study at any time. The findings of the study will be disseminated through peer-reviewed journals and national and international conference presentations and to the participants through communication within the dialysis network in which this study is conducted.

\section{BACKGROUND}

Cognitive impairment and major neurocognitive disorder (previously described as dementia) ${ }^{1}$ are frequently experienced by older adults and are associated with institutional care. ${ }^{2}$ Estimates suggest about one in six adults over 65 years of age meet the criteria for cognitive impairment without neurocognitive disorder and approximately $8 \%$ have neurocognitive disorder. ${ }^{3}$ In 2010, cognitive impairment was associated with 73 
million years lived with disability worldwide, and the disabling impact of cognitive impairment has shown a marked increase in the past two decades. ${ }^{4}$ Numerous pathologies can result in cognitive impairment and include those associated with Alzheimer's disease (neurofibrillary tangle and $\beta$-amyloid pathology), vascular disease, depression, medication, psychiatric illness, delirium and pre-existing intellectual disability. ${ }^{5}$ Documented cognitive impairment increases the risk of subsequent neurocognitive disorder; in people with cognitive impairment, approximately $5-10 \%$ develop neurocognitive disorder each year. ${ }^{6}$

Dialysis patients are at increased risks of cognitive impairment due to the higher prevalence of multimorbidity including cerebrovascular disease, depression, neuroactive medications and lower educational attainment than seen in the general older population. ${ }^{7}$ Consequently, people treated with haemodialysis are frequently affected by moderate-to-severe impairment of neurocognitive functioning ${ }^{8-10}$ that predicts poorer survival. ${ }^{11}{ }^{12}$ In a cross-sectional study involving 374 adults treated with haemodialysis, nearly three-quarters had moderate-tosevere cognitive impairment. ${ }^{8}$ However, while there are several existing studies of cognition in the dialysis setting, and an indication of frequent loss of executive functioning, ${ }^{13}$ a study with simultaneous assessment of numerous cognitive domains (executive function, perceptual-motor function, language, learning and memory, complex attention) in the dialysis setting has not been available to facilitate a detailed understanding of the specific cognitive deficits associated with end-stage kidney disease.

In addition, the associations of impaired neurocognitive function with levels of patient physical and social functioning-such as activities of daily living-remain uncertain, and are especially relevant to understanding the impact of cognitive functioning on dialysis patient-level experiences and outcomes. In a recent study involving 148 participants treated with haemodialysis or peritoneal dialysis, cognitive impairment (particularly executive function) was independently correlated with functional dependence in activities of daily living, although numbers of affected participants in this study was small and a comprehensive analysis of domains of cognitive function was limited. ${ }^{14}$

Additional large studies of the patterns and clinical impact of cognitive impairment in people with chronic kidney disease adjusted for age, gender and education would help provide robust measures of prevalence, evaluate the key clinical correlates of cognitive impairment controlling for sociodemographic and clinical variables, and further explore the associations of cognitive dysfunction with patient-relevant outcomes (including dependence in activities of daily living, cardiovascular and total mortality, institutionalisation, and withdrawal from treatment for end-stage kidney disease).

The COGNITIVE impairment in adults with end-stage kidney disease treated with HemoDialysis (COGNITIVEHD) study will evaluate-in adults with end-stage kidney disease treated with haemodialysis-the:
1. Presence and patterns of cognitive impairment in the following domains: executive function, perceptualmotor function, language, learning and memory, and complex attention.

2. Clinical significance of cognitive test status by comparing functional status of participants with and without cognitive deficits.

3. Association between cognitive impairment and cardiovascular and total mortality, major cardiovascular events, fatal and non-fatal myocardial infarction, fatal and non-fatal stroke, institutionalisation, and withdrawal from dialysis controlling for potentially confounding socioclinical factors.

We hypothesise that many dialysis patients will have clinically important cognitive impairment that is associated with specific deficits in their physical and social function. We also anticipate that cognitive impairment will be associated with specific clinical outcomes including survival, cardiovascular events, and withdrawal from treatment for end-stage kidney disease.

\section{METHODS AND ANALYSIS}

\section{Study design}

The COGNITIVE-HD study is a prospective, longitudinal cohort study (see figure 1 for flow diagram of study conduct). All 958 community-dwelling adults treated in a collaborative dialysis network in Italy were approached and invited to participate between July 2013 and April 2014. Of these, $751(78.4 \%)$ provided written and informed consent and were enrolled for assessments and study follow-up.

Consent forms had been approved by the relevant Human Research Ethics Committees for each investigational site before each clinic joined the study. A sample consent form and patient information sheet was provided to participating sites before study recruitment. Potential participants had an initial consultation with study personnel to discuss participation and were given an information sheet for the study. The participant or their representative gave written and informed consent before enrolment or completion of any study-specific procedures. Participants were asked to provide consent to data being obtained for the study using data linkages to centralised databases that contained additional demographic, clinical, biochemical and event (mortality and hospitalisation) data.

\section{Study population}

Adults were eligible for COGNITIVE-HD if they: (1) had end-stage kidney disease; (2) were treated with longterm outpatient haemodialysis for at least the previous 90 days; (3) were 18 years or older; (4) their treating team agreed to involvement in the study; and (5) were willing to provide written and informed consent. We excluded patients from COGNITIVE-HD if they: (1) were unable to participate in study procedures even if assisted; (2) had a life expectancy less than 6 months 


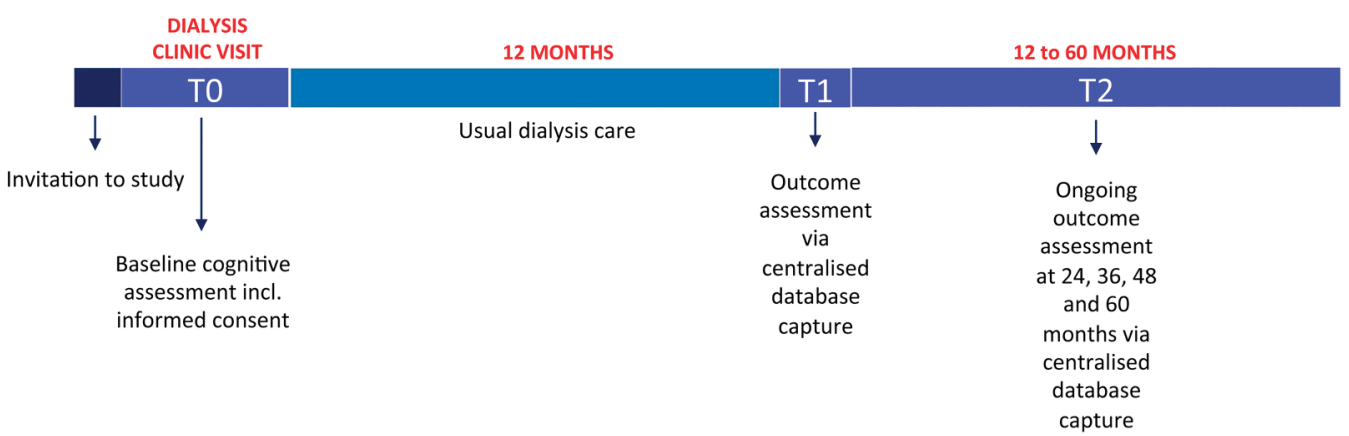

Figure 1 Study design.

according to their treating physician; (3) had a planned kidney transplantation within 6 months of baseline; or (4) had anticipated recovery of kidney function.

\section{Measures}

Table 1 gives an overview of the variables that were assessed at baseline and planned during follow-up in the study and the corresponding instruments used for assessment. The COGNITIVE-HD study used these measures to evaluate the following outcomes: (1) point prevalence of cognitive impairment; (2) characterisation of the pattern of cognitive deficits including executive function, perceptual-motor function, language, learning and memory, and complex attention; (3) clinical significance of cognitive test status by evaluating the functional status of participants with cognitive deficits versus those without deficit (Activities of Daily Living (ADL) and Instrumental Activities of Daily Living (IADL)); (4) association of cognitive impairment and neurocognitive disorder with patient-level outcomes including total and cause-specific mortality (cardiovascular and infection), major cardiovascular events, fatal and non-fatal myocardial infarction, fatal and non-fatal stroke, institutionalisation, and withdrawal from dialysis, controlled for clinical, demographic and dialysis-related variables.

Research staff at each centre attended an initial training session at the central study centre (Bari, Italy) before the study started. These investigators trained local staff according to standard operating procedures. Staff conducting the neuropsychological examination were trained centrally and certified.

Cognitive testing was carried out by native Italian speakers and all participants were native speakers of the Italian language. All tests were performed during haemodialysis treatment. The neurocognitive testing was done by a neuropsychologist within $1 \mathrm{~h}$ after the start of dialysis treatment. Participants with sensory (visual and hearing) or motor impairment did not complete the tests that required these senses for completion. Testing took $30 \mathrm{~min}$ on average. Following neurocognitive testing, participants completed questionnaires about affect and personality as described below.

\section{BASELINE DEMOGRAPHIC AND CLINICAL DATA}

Demographic, clinical, laboratory and dialysis-related data were obtained from a centralised database within 1 month of enrolment using a standardised identification code to link study procedures to database information. Standardised baseline data included age, sex, race, clinic, education, marital and occupational status, family income, financial stress, housing, alcohol intake, smoking history, physical activity, menopausal status, body mass index, protein catabolic rate, cause of kidney disease, cardiovascular comorbidity including stroke, diabetes, hypertension, medication prescription, dialysis prescription, and serum levels of haemoglobin, phosphorus, parathyroid hormone, calcium, ferritin, albumin and total cholesterol.

\section{STUDY FACTORS}

\section{Neuropsychological assessment}

At baseline, each participant underwent a standard neuropsychological battery composed of one global cognitive task and eight tests specific to cognitive domains (table 1). Some tests listed in the table 1 explore more than one neurocognitive domain; therefore, the predominant neurocognitive domain being tested is shown. The full details of the tests used to assess cognitive functioning tests used were as follows:

1. Mini-Mental State Examination ${ }^{15}$ (MMSE) to assess the global cognition. The MMSE is a 30-point administered questionnaire including orientation, immediate and short-term recall, attention and calculation word finding, figure construction, reading and writing skills, and the ability to follow a three-step command. Norms specific to age and education (in years) in an Italian population are available ${ }^{16}$ and a higher number of items indicates better cognitive function.

2. The Rey Auditory Verbal Learning Test (RAVLT) to evaluate verbal episodic memory. The RAVLT requires the immediate free recall of the same list of 15 unrelated words over five trials, followed by a delayed recall and recognition task. Delayed recall is then examined with a recall of list after a 15 min delay without previous presentation. The recognition task includes distractor and target words; participants had to indicate whether the presented word belongs to the learned list or not.

3. The Symbol Digit Modalities Test (SDMT) ${ }^{17}$ and Digit Span Forward (DSF) to assess attention components.

A. SDMT was used to measure sustained attention and information processing speed. In the oral 
Table 1 Summary of assessments and testing instruments used in the COGNITIVE-HD study

\begin{tabular}{|c|c|c|c|c|}
\hline & Instrument/assessment tool & Baseline & 12 months & 24 months \\
\hline \multicolumn{5}{|l|}{ Cognitive functioning } \\
\hline Executive function & $\begin{array}{l}\text { Categorical verbal fluency (fruit and } \\
\text { vegetables) }{ }^{18}\end{array}$ & $\mathrm{X}$ & & \\
\hline Planning & Letter fluency (FAS) ${ }^{18}$ & & & \\
\hline \multicolumn{5}{|l|}{ Decision-making } \\
\hline \multicolumn{5}{|l|}{ Working memory } \\
\hline \multicolumn{5}{|l|}{ Responding to feedback } \\
\hline \multicolumn{5}{|l|}{ Inhibition } \\
\hline \multicolumn{5}{|l|}{ Flexibility } \\
\hline Perceptual-motor function & Figure copying ${ }^{28}$ & $X$ & & \\
\hline Visual perception & Line orientation ${ }^{29}$ & & & \\
\hline \multicolumn{5}{|l|}{ Visuoconstructional reasoning } \\
\hline \multicolumn{5}{|l|}{ Perceptual-motor coordination } \\
\hline Language & RBANS Picture Naming subtest ${ }^{30}$ & $X$ & & \\
\hline \multicolumn{5}{|l|}{ Object naming } \\
\hline \multicolumn{5}{|l|}{ Word finding } \\
\hline \multicolumn{5}{|l|}{ Fluency } \\
\hline \multicolumn{5}{|l|}{ Grammar and syntax } \\
\hline \multicolumn{5}{|l|}{ Receptive language } \\
\hline Learning and memory & RAVLT Immediate Recall ${ }^{31}$ & $\mathrm{X}$ & & \\
\hline Free recall & RAVLT Delayed Recall ${ }^{31}$ & & & \\
\hline Cued recall & RAVLT Recognition & & & \\
\hline \multicolumn{5}{|l|}{ Recognition memory } \\
\hline \multicolumn{5}{|l|}{$\begin{array}{l}\text { Semantic and autobiographical long-term } \\
\text { memory }\end{array}$} \\
\hline \multicolumn{5}{|l|}{ Implicit learning } \\
\hline Complex attention & Digit span (forward) ${ }^{19}$ & $X$ & & \\
\hline Sustained attention & Digit span (backward) ${ }^{19}$ & & & \\
\hline Divided attention & $\begin{array}{l}\text { Symbol Digit Modality Test (oral } \\
\text { version) })^{17}\end{array}$ & & & \\
\hline \multicolumn{5}{|l|}{ Selective attention } \\
\hline \multicolumn{5}{|l|}{ Processing speed } \\
\hline Mini-Mental State Examination & Mini-Mental State Examination ${ }^{15}$ & $\mathrm{X}$ & & \\
\hline Depression & HADS-D 20 & $\mathrm{X}$ & & \\
\hline Anxiety & HADS-A 20 & $x$ & & \\
\hline \multirow[t]{2}{*}{ Activities of daily living } & $\mathrm{ADL}^{25}$ & $x$ & & \\
\hline & $\mathrm{IADL}^{25}$ & & & \\
\hline General psychological functioning & MCMI-III 2223 & $\mathrm{X}$ & & \\
\hline \multicolumn{5}{|l|}{ Clinical outcomes } \\
\hline All-cause mortality & Centralised clinical registry & & $\mathrm{X}$ & $\mathrm{X}$ \\
\hline Cardiovascular mortality & & & $X$ & $\mathrm{X}$ \\
\hline Infection-related mortality & & & $x$ & $\mathrm{X}$ \\
\hline Major cardiovascular events & & & $x$ & $\mathrm{X}$ \\
\hline Fatal or non-fatal myocardial infarction & & & $\mathrm{X}$ & $\mathrm{X}$ \\
\hline Fatal or non-fatal stroke & & & $\mathrm{X}$ & $X$ \\
\hline Withdrawal from treatment & & & $\mathrm{X}$ & $\mathrm{X}$ \\
\hline Institutionalisation & & & $x$ & $x$ \\
\hline
\end{tabular}

ADL, Activities of Daily Living; COGNITIVE-HD, COGNITIVE impairment in adults with end-stage kidney disease treated with Hemo Dialysis; FAS, Phonemic Fluency Test; HADS, Hospital Anxiety and Depression Scale; IADL, Instrumental Activities of Daily Living; MCMI, Millon Clinical Multiaxial Inventory; RAVLT, Rey auditory verbal learning test; RBANS, Repeatable Battery for the Assessment of Neuropsychological Status.

version, the participant was given a sheet of paper at the top of which was printed the key (9 abstract symbols matched to 9 corresponding numbers). The participant was asked to make as many of 110 associations within $90 \mathrm{~s}$ and reported the associations orally to the researcher. The possible scores were between 0 and 110 ;
B. DSF was used to measure the attentional capacity (processing speed and short-term memory). Participants were presented with a list of digits and was asked to repeat the list in the same order. The length of the digit sequences gradually increased, starting with a sequence of three numbers to a sequence of maximum nine. The span is established as the 
length of the longest list recalled correctly. The possible scores were between 0 and 9 .

4. The Phonemic Fluency Test (FAS) ${ }^{18}$ and Digit Span Backward (DSB) to assess the executive function.

A. FAS is a phonological task of word finding which requires a executive search strategy. In this test, participants were asked to generate as many words out loud as possible beginning with ' $\mathrm{F}$ ', 'A', 'S' in $1 \mathrm{~min}$ for each category. A higher number of items indicates better cognitive function.

B. $\mathrm{DSB}^{19}$ was used to measure verbal working memory. The procedure is the same as the DSF, except that in this case participants have to reproduce the sequence of digits in the reverse order and the longest list consists of eight items.

5. Repeatable Battery for the Assessment of Neuropsychological Status (RBANS) Semantic Fluency (RBANS-SF) and Picture Naming (RBANSPN) subtests to assess language production abilities.

A. RBANS-SF evaluates semantic fluency. The researcher asked participants to say out loud as many names of vegetables and fruits as possible within $1 \mathrm{~min}$. A higher number of items indicates better cognitive function.

B. RBANS-PN: In this test which assesses naming, the participant was asked to name familiar objects shown in 10 line drawings and given 1 point for each correctly named picture to provide a total score out of 10 . The procedure provides semantic cues when there are naming difficulties.

6. RBANS Figure Copy and RBANS Line Orientation subtests to evaluate visuospatial abilities.

A. RBANS Figure Copy evaluates organisational and visuoconstructional abilities. Participants were asked to copy a specified complex figure onto a piece of paper with the figure used as a reference. The participant was given as much time as needed to complete the task. Ten major figural elements were identified in the drawing and each figural element was scored as 2 points if the element was drawn accurately and placed correctly in the figure (1 point for accuracy and 1 point for placement). The total possible score was 20 .

B. RBANS Line Orientation measures the visuospatial orientation. Participants were asked to match a pair of angled lines on a stimulus card to 2 of 13 numbered lines appearing on a reference card. Each correct response was given one credit and the total number of correct answers out of 10 was recorded. The total possible score was 20 .

The raw scores on cognitive tests were converted to standardised scores (ie, z-scores) based on Italian normative data. Scores will be summed and averaged to derive composite scores within each domain and a global index of neuropsychological dysfunction.

The presence of neurocognitive impairment is defined as 1.5 SD below the mean for healthy agematched normative data.

\section{Behavioural rating scales}

Participants completed a comprehensive series of behaviour rating inventories to survey a broad range of emotional behaviour using the following questionnaire:

1. Hospital Anxiety and Depression Scale (HADS) $:{ }^{20}$ The HADS is a self-report scale to assess anxiety (HADS-A) and depression (HADS-D) in the context of physical health problems, to minimise the impact of diseasespecific somatic symptoms of chronic disease such as fatigue or sleep disturbance. The HADS consisting of 14 multiple choice (0-3 Likert scale) items probing symptoms of anxiety ( 7 items) and depression (7 items). Anxiety (HADS-A) and depression (HADS-D) scores range from 0 (no symptoms) to 21 (most severe symptoms). Cut-off scores of 10 or above are considered an accurate indicator of major depression (HADS-D) or generalised anxiety disorder (HADS-A). We used a cut-off score of $\geq 7$ for the depression subscale (HADS-D) and $\geq 6$ for the anxiety subscale (HADS-A) to indicate presence of depression and anxiety in the setting of end-stage kidney disease. ${ }^{21}$

2. Starkstein Apathy Scale (SAS). The SAS is designed to assess cognitive, behaviour and emotional aspects of goal-directed behaviour. This scale has 14 items, each rating between 0 and 3 . The possible total scores were between 0 and 42. Apathy was defined as a SAS score $>14$.

3. Millon Clinical Multiaxial Inventory-III (MCMIIII) $:^{22} 23$ The MCMI-III provides objective assessment of axis I clinical symptoms of and axis II personality disorders according to Diagnostic and Statistical Manual of Mental Disorders fourth edition (DSM-IV (American Psychiatric Association, APA), 2000). ${ }^{24}$ The MCMI-III is composed of 175 true-false questions to assess 14 personality scales (schizoid, avoidant, depressive, dependent, histrionic, narcissistic, antisocial, sadistic, compulsive, negativistic (passiveaggressive), masochistic (self-defeating), schizotypal, borderline, paranoid), 10 clinical syndrome scales (anxiety, somatoform, bipolar: mania, dysthymic, alcohol dependence, drug dependence, posttraumatic stress disorder; major depression, delusional disorder) and correction scales (modifying indices, random response indicators and Grossman Facet Scales). The scales are scored using Base Rate (BR) scores based on the prevalence of any personality pattern or clinical syndrome in the target population. A BR score of 75 is assumed to reflect presence of some features of a given personality pattern or clinical syndrome, and a BR score of 85 is assumed to indicate definite presence of the personality pattern or clinical syndrome. 


\section{OUTCOMES}

\section{Activities of daily living}

The following tools were used to assess activities of daily living:

1. Activities of Daily Living: ${ }^{25}$ The ADL Scale assessed 'self-maintaining' activities of daily living including toileting, feeding, dressing, grooming, physical ambulation and bathing. The ADL Scale was assessed by researchers and contained six items that were rated with a summary score from 0 (low functioning) to 6 (high functioning).

2. Instrument Activities of Daily Living: ${ }^{25}$ The IADL Scale assessed complex activities (termed 'instrumental activities of daily living') that are needed to function in a community setting (using a telephone, food preparation, housekeeping, laundry, transportation, responsibility for medications and managing finances). The capacity for an individual to complete these complex functions is normally lost before the more basic activities of daily living (eg, bathing, toileting, eating). The IADL Scale was administered by researchers and contained eight items that were rated with a summary score from 0 (low functioning) to 8 (high functioning).

\section{Clinical outcomes}

After baseline cognitive evaluation, we will measure clinical outcomes using linked registry data at 12 months of study follow-up. Data for all patient-level outcomes including total mortality and cause-specific mortality (cardiovascular and infection), major cardiovascular events, fatal and non-fatal myocardial infarction, fatal and nonfatal stroke, institutionalisation, and withdrawal from treatment will be obtained through data linkages to a centralised database. In this database, every change in participant status is updated on a monthly basis by a managing clinician who is unaware of the cognitive function test scores. A cardiovascular-related death or hospitalisation includes death or hospitalisation attributed to acute myocardial infarction, pericarditis, atherosclerotic heart disease, cardiomyopathy, cardiac arrhythmia, cardiac arrest, valvular heart disease, pulmonary oedema, congestive cardiac failure, cerebrovascular accident including intracranial haemorrhage, ischaemic brain damage including anoxic encephalopathy, or mesenteric infarction or ischaemic bowel. An infection-related death includes septicaemia due to internal vascular access, central nervous system infection (brain abscess, meningitis, encephalitis), septicaemia due to peripheral vascular disease or gangrene, cardiac infection (endocarditis), pulmonary infection (pneumonia or influenza), abdominal infection (peritonitis, perforated bowel, diverticular disease, gallbladder infection), or genitourinary infection (urinary tract infection, pyelonephritis, renal abscess).

\section{PLANNED STATISTICAL ANALYSES}

The initial data analysis will be descriptive. Participant baseline characteristics (clinic, demographics, clinical characteristics, dialysis treatment, etc) will be described using frequencies for categorical variables and mean, median, range, SD for continuous variables. To assess for the presence and patterns of cognitive impairment, we will calculate the score of the individual cognitive tests on each of the items from the neuropsychological battery for each participant (table 1). We will then compare the age-adjusted and education-adjusted individual cognitive test results against age-adjusted and education-adjusted published norms, preferably from Italian populations for tests with language components when these are available. The z-scores for each test score will be displayed graphically against corresponding population norms to identify and show specific patterns of cognitive function in the study population. ${ }^{26}$

For each test, we will then use a cut-point of 1.5 SDs below the normal population mean score to indicate impairment in the cognitive test for individual participants. We will then group the cognitive tests into the prespecified domains described in table 1 and classify the cognitive function domains as impaired or not (relative to corresponding population norms) if one or more of the tests are below the population norm. We will then use the DSM fifth edition ${ }^{1}$ criteria to classify participants as having cognitive impairment or not. As our testing battery has limitations, partly due to time and potential for participant fatigue with a wider range of tests, we will use a principal component analysis in secondary analyses to explore the possibility of generating a composite cognitive measure for people with end-stage kidney disease treated with dialysis.

We will use univariate and multivariate analyses to compare the clinical and demographic characteristics in participants with and without cognitive impairment as well as correlates of impairment in specific cognitive domains. To assess the association between cognitive impairment and activities of daily living, we will compare ADL and IADL scores between those participants classified as having cognitive impairment versus others using a two-sample t test.

To evaluate the associations between cognitive impairment and clinical outcomes, we will conduct random-effects Cox proportional hazards analysis adjusted for potential confounding variables and clustered by treatment clinic to account for the intracluster correlation of baseline characteristics and mortality risk within clinics. We will check for confounders, interactions and multicollinearity among independent variables. The final models will be adjusted for all confounders and baseline covariates judged to have clinical importance. We will also consider Cox proportional hazards regression analysis in participants matched on their propensity score for cognitive impairment, considering the following covariables-age, sex, education, previous cardiovascular disease, duration of dialysis treatment, anaemia, blood pressure and psychoactive medication.

Missing data, not missing at random, will be addressed using standard multiple imputation techniques as described by Rubin. ${ }^{27}$ 
The significance level will be set at 0.05 . All analyses will be performed using SAS Language (Release V.9.4; http://www.sas.com). Participants will be censored within survival analyses if they emigrate from the dialysis network, are transplanted or experience recovery of their kidney function. We will conduct sensitivity analyses excluding participants with additional psychiatric diagnoses (based on the MCMI) and those with HADS scores consistent with clinical anxiety or depression.

\section{SAMPLE SIZE AND POWER CALCULATION}

A sample of 750 participants was estimated to be necessary to provide a statistical power of $83 \%$ for detecting a relative risk of 1.7 for mortality associated with cognitive impairment, with a mortality rate of $13 \%$ in the control group, a $5 \%$ significance level and a $25 \%$ prevalence of cognitive impairment.

\section{ETHICS AND DISSEMINATION \\ Ethical considerations}

The COGNITIVE-HD study is based on written and informed consent. No study conduct will be carried out before written and informed consent is provided. Participants can withdraw from the study at any point in time without any impact on their clinical care. Clinical care will be provided throughout the study according to standardised local procedures within the dialysis network. Study data will be managed confidentially and anonymously.

\section{Dissemination}

The findings of the study will be disseminated through peer-reviewed journals, national and international conference presentations and to the participants through communication within the dialysis network in which this study is conducted via a regular newsletter.

\section{DISCUSSION}

We have designed the COGNITIVE-HD study to evaluate the prevalence and patterns of cognitive impairment in the setting of end-stage kidney disease, adjusting for age, gender and education in adults with end-stage kidney disease treated with haemodialysis. The COGNITIVEHD will also assess the association of cognitive impairment with activities of daily living and clinical outcomes including hospitalisation, institutionalisation and mortality. This study will generate potential testable targets for evaluation in pragmatic multicentre trials and prospective meta-analyses.

Our study design, while incorporating data from several centres and using validated and robust neurocognitive assessment, has potential limitations. To ensure sufficient data from a broad range of participants, we have used a sample of clinics within a single country (Italy) to maximise recruitment. This may limit external validity in other dialysis populations but will still be the largest complete neurocognitive assessment of adults treated with haemodialysis to date. Mortality and other end point data will be obtained using linkages to a data registry; there will not be adjudication of clinical end points by personnel blinded to exposure and there may be some misclassification of clinical outcomes.

\section{CONCLUSION}

In conclusion, COGNITIVE-HD has the potential to inform large pragmatic trials of cognitive impairment interventions in the setting of advanced kidney disease. Existing effective strategies to improve health outcomes in this specific population are scarce and urgently needed.

\section{Author affiliations}

${ }^{1}$ Department of Medicine, University of Otago Christchurch, Christchurch, New Zealand

${ }^{2}$ Diaverum Medical Scientific Office, Lund, Sweden

${ }^{3}$ Division of Nephrology and Transplantation, Amedeo Avogadro University of Eastern Piedmont, Novara, Italy

${ }^{4}$ Neurodegenerative Diseases Unit, Department of Clinical Research in

Neurology, University of Bari "A. Moro", Tricase, Lecce, Italy

${ }^{5}$ Huntington Disease Service, Westmead Hospital, Westmead, NSW, Australia

${ }^{6}$ Sydney School of Public Health, University of Sydney, Sydney, NSW,

Australia

${ }^{7}$ Department of Nephrology, Princess Alexandra Hospital, Woolloongabba,

Australia

${ }^{8}$ Cumming School of Medicine, Health Services, University of Calgary, Calgary, Alberta, Canada

${ }^{9}$ Department of Basic Medical Sciences, Neurosciences and Sense Organs, University of Bari "A. Moro", Bari, Italy

${ }^{10}$ Department of Emergency and Organ Transplantation, University of Bari, Piazza Giulio Cesare, Bari, Italy

${ }^{11}$ Diaverum Academy, Bari, Italy

Acknowledgements The authors wish to thank Professor Perminder Sachdev for helpful advice during the preparation of the manuscript.

Collaborators Puglia region (Brindisi territory): AV Cagnazzo, R Antinoro, ML Sambati, C Donatelli, N Dambrosio, C Saturno, A Marangelli, F Pedone, G Matera, M Benevento, S Papagni, F Alicino; Sicily region (Catania territory): $G$ Latassa, A Molino, F Grippaldi, D Bertino, G Montalto, S Messina, S Campo, P Nasisi, A Failla, A Bua, S Pagano, G Marino, AMM, N Sanfilippo, D Rallo, A Maniscalco, C Capostagno, G Randazzo, M Fici, A Lupo, R Fichera, A D'angelo; Lazio region (Roma territory): R Di Toro Mammarella, M Meconizzi, E Boccia, M Mantuano, A Flammini, L Moscardelli.

Contributors SCP, GL and GFMS conceived and designed this study and drafted the manuscript. MR, MRB, AI, VS, PN, LG, AMM, AvZ, GW, RT, JCC, DWJ, MT, JH, CW and GL contributed to the design of the study and reviewed the manuscript for intellectual content. GFMS obtained funding.

Funding This work was supported by funding from Diaverum $A B$ for the conduct of this study.

Competing interests MR, VS, PN, LG, JH, CW and GFMS are employees of Diaverum. SCP receives a Rutherford Discovery Fellowship from the New Zealand Royal Society.

Ethics approval COGNITIVE-HD has received ethics approval from the responsible Human Research Ethics Committees in the involved units in Italy. This protocol was approved before study conduct by the following responsible ethics committees: Catania (approval reference 186/BE; 26/09/2013),

Agrigento (protocol numbers 61-62; 28/6/2013), USL Roma C (CE 39217; 24/6/2013) USL Roma F (protocol number 0041708; 23/7/2013), USL Latina (protocol number 20090/A001/2011; 12/7/2013), Trapani (protocol number 3413; 16/7/2013), Brindisi (protocol number 40259; 6/6/2013) and Trapani 
(protocol number 50; 5/7/2013). The findings of the study will be disseminated through peer-reviewed journals, national and international conference presentations and to the participants through communication within the dialysis network in which this study is conducted.

Provenance and peer review Not commissioned; externally peer reviewed.

Open Access This is an Open Access article distributed in accordance with the Creative Commons Attribution Non Commercial (CC BY-NC 4.0) license, which permits others to distribute, remix, adapt, build upon this work noncommercially, and license their derivative works on different terms, provided the original work is properly cited and the use is non-commercial. See: http:// creativecommons.org/licenses/by-nc/4.0/

\section{REFERENCES}

1. American Psychiatric Association. Diagnostic and statistical manual of mental disorders. 5th edn. Washington DC, 2013.

2. Graham JE, Rockwood K, Beattie BL, et al. Prevalence and severity of cognitive impairment with and without dementia in an elderly population. Lancet 1997;349:1793-6.

3. Brookmeyer R, Gray S, Kawas C. Projections of Alzheimer's disease in the United States and the public health impact of delaying disease onset. Am J Public Health 1998;88:1337-42.

4. Murray CJ, Vos T, Lozano R, et al. Disability-adjusted life years (DALYs) for 291 diseases and injuries in 21 regions, 1990-2010: a systematic analysis for the Global Burden of Disease Study 2010. Lancet 2012;380:2197-223.

5. DeCarli C. Mild cognitive impairment: prevalence, prognosis, aetiology, and treatment. Lancet Neurol 2003;2:15-21.

6. Morris JC, Storandt M, Miller JP, et al. Mild cognitive impairment represents early-stage Alzheimer disease. Arch Neurol 2001;58:397-405.

7. Sarnak MJ, Tighiouart H, Scott TM, et al. Frequency of and risk factors for poor cognitive performance in hemodialysis patients. Neurology 2013;80:471-80.

8. Murray AM, Tupper DE, Knopman DS, et al. Cognitive impairment in hemodialysis patients is common. Neurology 2006;67:216-23.

9. Sehgal AR, Grey SF, DeOreo PB, et al. Prevalence, recognition, and implications of mental impairment among hemodialysis patients. Am $J$ Kidney Dis 1997;30:41-9.

10. Brady CB, Gaziano JM, Cxypoliski RA, et al. Homocysteine lowering and cognition in CKD: the Veterans Affairs homocysteine study. Am $J$ Kidney Dis 2009;54:440-9.

11. Griva K, Stygall J, Hankins M, et al. Cognitive impairment and 7 -year mortality in dialysis patients. Am J Kidney Dis 2010;56:693-703.

12. Drew DA, Weiner DE, Tighiouart $\mathrm{H}$, et al. Cognitive function and all-cause mortality in maintenance hemodialysis patients. $A m \mathrm{~J}$ Kidney Dis 2015;65:303-11

13. Kurella Tamura M, Larive $\mathrm{B}$, Unruh $\mathrm{ML}$, et al. Prevalence and correlates of cognitive impairment in hemodialysis patients: the Frequent Hemodialysis Network trials. Clin J Am Soc Nephrol 2010;5:1429-38.

14. Kavanagh NT, Schiller B, Saxena AB, et al. Prevalence and correlates of functional dependence among maintenance dialysis patients. Hemodial Int 2015;19:593-600.

15. Folstein MF, Folstein SE, McHugh PR. "Mini-mental state": a practical method for grading the cognitive state of patients for the clinician. J Psychiatr Res 1975;12:189-98.

16. Magni E, Binetti G, Bianchetti A, et al. Mini-Mental State Examination: a normative study in Italian elderly population. Eur $J$ Neurol 1996;3:198-202.

17. Smith A. The Symbol-Digit Modalities Test: a neuropsychologic test for economic screening of learning and other cerebral disorders. Learn Disord 1968;3:83-91.

18. Lezak MD. Neuropsychological assessment. Oxford, Oxfordshire: Oxford University Press, 1995.

19. Kaufman A, Lichtenberger E. Assessing adolescent and adult intelligence. 3rd edn: Hoboken, NJ: Wiley, 2006.

20. Zigmond A, Snaith R. The hospital anxiety and depression scale. Acta Psychiatr Scand 1983;67:361-70.

21. Preljevic VT, Østhus TB, Sandvik L, et al. Screening for anxiety and depression in dialysis patients: comparison of the Hospital Anxiety and Depression Scale and the Beck Depression Inventory. J Psychosom Res 2012;73:139-44.

22. Millon T. The Millon inventories: clinical and personality assessment In: Millon Theodore, ed. New York, NY, US: Guilford Press, 1997:xiii 553.

23. Millon T, Davis R. The MCMI-III: present and future directions. $J$ Pers Assess 1997;68:69-85.

24. American Psychiatric Association. Diagnostic and statistical manual of mental disorders (4th edition revised). 2000. doi:10.1176/appi. books.9780890423349.

25. Lawton MP, Brody EM. Assessment of older people: self-maintaining and instrumental activities of daily living. Gerontologist 1969;9:179-86.

26. Reid WG, Hely MA, Morris JG, et al. Dementia in Parkinson's disease: a 20-year neuropsychological study (Sydney Multicentre Study). J Neurol Neurosurg Psychiatry 2011;82:1033-7.

27. Rubin D. Multiple imputation for nonresponse in surveys. New York: J Wiley \& Sons, 1987.

28. Osterrieth P. Filetest de copie d'une figure complex: Contribution a l'etude de la perception et de la memoire [The test of copying a complex figure: a contribution to the study of perception and memory. Arch Psychol 1944;30:286-356.

29. Grant I, Adams K. Neuropsychological assessment of neuropsychiatric and neuromedical disorders. Oxford University Press, 2009.

30. Randolph C, Tierney M, Mohr E, et al. The Repeatable Battery for the Assessment of Neuropsychological Status (RBANS): preliminary clinical validity. J Clin Exp Neuropsychol 1998;20:310-19.

31. Carlesimo G, Caltagirone C, Gainotti G. The Mental Deterioration Battery: normative data, diagnostic reliability and qualitative analyses of cognitive impairment. The Group for the Standardization of the Mental Deterioration Battery. Eur Neurol 1996;36:378-84. 